Check for updates

Cite this: RSC Adv., 2017, 7, 56537

\title{
A postsynthetic ion exchange method for tunable doping of hydroxyapatite nanocrystals $\uparrow$
}

\author{
Gaoyu Chen, $t^{\mathrm{ab}}$ Xiaoyan Zheng,,$^{\mathrm{a}}$ Chong Wang, ${ }^{\mathrm{b}}$ Junfeng Hui, ${ }^{a}$ Xuexi Sheng, ${ }^{\mathrm{b}}$ \\ Xiangxing Xu, (D) ${ }^{* b}$ Jianchun Bao, (D) ${ }^{b}$ Weijun Xiu, ${ }^{c}$ Lihui Yuwen ${ }^{\star c}$ and Daidi Fan ${ }^{* a}$
}

Hydroxyapatite (HAp) is the main inorganic component of human bones and teeth. The doping of HAp nanocrystals plays an important role in tissue engineering, drug delivery, biomarkers and artificial bones. In this article, a postsynthetic metal ion exchange method was developed for doping hydroxyapatite (HAp) nanocrystals in organic solutions. It can be mono- or multi-ion doped with $\mathrm{Mg}^{2+}, \mathrm{Sr}^{2+}, \mathrm{Zn}^{2+}, \mathrm{Mn}^{2+}$, $\mathrm{Fe}^{3+}$ or $\mathrm{Cu}^{2+}$ etc., despite the significant radius variation of $\mathrm{Ca}^{2+}$ and doping ions. The doping ratio can be tuned in a wide range, e.g. $\mathrm{Fe}^{3+}$ of $0-20 \%$, which is much higher than the ion exchange performed in aqueous solution. The structure and morphology of the HAp nanocrystals were preserved after postsynthetic doping, suggesting potential biological applications.

Received 22nd September 2017 Accepted 8th December 2017

DOI: 10.1039/c7ra10516a

rsc.li/rsc-advances
$\mathrm{Fe}^{3+/ 2+}$ not only exists in the blood, but also in bones and enamel; rare earth ions can serve as fluorescent centers and so on. Therefore, understanding the doping mechanisms is of fundamental importance in developing nanomaterials with desired functionalities.

To date, various chemical doping strategies have been developed for nanocrystals, ${ }^{19,20}$ e.g. co-nucleation doping, ${ }^{21}$ growth doping, ${ }^{22}$ dopant-nucleation, ${ }^{23}$ diffusion doping, ${ }^{24}$ ionexchange doping, ${ }^{25}$ etc. Among them, the most widely adopted method is the co-nucleation doping. It features in the dopant and host precursors being loaded into the synthesis system simultaneously at the beginning of the reaction, where the doping and nucleation steps could not be decoupled and the doping continues during the following growth stage. Although there has been successfully HAp doping examples via this method, ${ }^{9-18}$ there remains some critical challenges. First, the doping ions would affect the nucleation and growth kinetics or crystallization dynamics, leading to the change of the final morphology of the nanocrystals. Therefore, on one hand, the size and shape of the nanocrystals may be tuned by the concentration of the doping metal ions; on the other hand, it sets up an obstacle toward HAp nanocrystals of the same morphology and size with different doping ratios. Second, the doping ions may form impurity phases during the growth of HAp nanocrystals. Third, the ion exchange of HAp nanocrystals performed in aqueous containing solutions requires the $\mathrm{PH}$ value near neutral or alkaline, for the HAp may decompose in acidic solution. ${ }^{26-29}$ However, many salts in water exhibit acidity because of their hydrolysis, such as $\mathrm{FeCl}_{3}$ and $\mathrm{CuCl}_{2}$, etc. The last but not the least, only the ionic radius of the cation falls within $0.09-0.13 \mathrm{~nm}$, approaching that of $\mathrm{Ca}^{2+}$ of $0.1 \mathrm{~nm}$, the $\mathrm{Ca}^{2+}$ can be easily replaced to form heavy doped HAps. ${ }^{30}$ For examples, there are many reports on Sr-HAp with the doping 
ratio exceeding $50 \%$, and even $100 \%$ Sr-HAp was reported, for the radius of $\mathrm{Sr}^{2+}$ is $0.12 \mathrm{~nm} ;^{31}$ The radius of $\mathrm{Mg}^{2+}$ is $0.072 \mathrm{~nm}$, $10 \%$ is believed to be the upper limit; ${ }^{32}$ the radius of $\mathrm{Fe}^{3+}$ is even smaller of $0.055 \mathrm{~nm}$, thus rarely $>5 \% \mathrm{Fe}^{3+}$ doped HAp was reported. ${ }^{33}$ All these limitations will affect the structure stability, ion releasing and thus the bio-properties of the doped HAp nanocrystals in service.

To solve the above challenges, we developed a postsynthetic strategy to doping HAp nanocrystals in non-aqueous organic solution. It was demonstrated that with this route, the doping ratio can be feasibly tuned by the concentration, temperature and time. It can be mono- or multi-metal ion doping. The doped HAp nanocrystals was highly pure without byproduct impurities. The morphology and size of the doped HAp nanocrystals were almost the same as the undoped ones. It is a practical route toward heavy doping which can hardly be achieved in aqueous ion exchange because of the unwanted hydrolysis and acidity. The doping ratio of $\mathrm{Fe}^{3+}$ can easily boost up to $>20 \%$. Also, the as-described method can make metal ions doped into the HAp crystal more effectively without destroying the crystal structure.

\section{Experimental section}

\subsection{Materials}

$\mathrm{Ca}\left(\mathrm{NO}_{3}\right)_{2} \cdot 4 \mathrm{H}_{2} \mathrm{O} \quad(\mathrm{AR},>99 \%), \quad \mathrm{Na}_{3} \mathrm{PO}_{4} \cdot 12 \mathrm{H}_{2} \mathrm{O} \quad(\mathrm{AR},>98 \%)$, $\mathrm{FeCl}_{3} \cdot 6 \mathrm{H}_{2} \mathrm{O}(\mathrm{AR}, \geq 99 \%), \mathrm{C}_{18} \mathrm{H}_{33} \mathrm{NaO}_{2}(\mathrm{CP},>98 \%), \mathrm{CuCl}_{2} \cdot 3 \mathrm{H}_{2} \mathrm{O}$ $(\mathrm{AR}, \geq 99 \%), \mathrm{Mn}\left(\mathrm{NO}_{3}\right)_{2} \cdot 4 \mathrm{H}_{2} \mathrm{O}(\mathrm{AR}, \geq 97.5 \%), \mathrm{Fe}\left(\mathrm{NO}_{3}\right)_{3} \cdot 9 \mathrm{H}_{2} \mathrm{O}$ $(\mathrm{AR}, \geq 98.5 \%), \mathrm{Sr}\left(\mathrm{NO}_{3}\right)_{2}(\mathrm{AR}, \geq 99.5 \%), \mathrm{Zn}\left(\mathrm{NO}_{3}\right)_{2} \cdot 6 \mathrm{H}_{2} \mathrm{O}$ (AR, $\geq 99 \%$ ), $\mathrm{Mg}\left(\mathrm{NO}_{3}\right)_{2} \cdot 6 \mathrm{H}_{2} \mathrm{O}(\mathrm{AR}, \geq 99 \%), \mathrm{NaIO}_{4}(\mathrm{AR}, \geq 99.5 \%)$, $\mathrm{Na}_{2} \mathrm{CO}_{3}$ (AR, >99.8\%), PEG20000 (AR, >90\%), ethyl acetate (AR, $\geq 99.5 \%$ ), acetonitrile (AR, $\geq 99.8 \%$ ), hexane (AR, $\geq 97 \%$ ), ethanol (AR, $\geq 99.7 \%$ ) and cyclohexane (AR, $\geq 99.7 \%$ ) were obtained from Sinopharm Chemical Reagent Co. The oleic acid (OA, 90\%) was purchased from Alfa Aesar. 1-Octadecene (ODE, 90\%) and oleylamine (OLA, 90\%), octadecylamine (ODA, $\geq 97.0 \%$ ) were purchased from Aldrich. All chemicals were used as received without further purification.

\subsection{Synthesis of oleate complex}

The metal-oleate complex was synthesis by reacting metal-salt and sodium oleate. In a typical synthesis of iron-oleate, $2.6 \mathrm{~g}$ ferric chloride was dissolved in $4 \mathrm{~mL}$ deionized water, $7.3 \mathrm{~g}$ sodium oleate was dissolved in the mixture of $12 \mathrm{~mL}$ deionized water and $16 \mathrm{~mL}$ ethanol, then they were mixed together in a flask and $32 \mathrm{~mL}$ hexane was put in at the same time, the resulting reddish brown mixture was heated to $65{ }^{\circ} \mathrm{C}$ for four hours, and cooled to room temperature, then the supernatant organic layer containing the iron-oleate complex was washed three times with $30 \mathrm{~mL}$ distilled water to remove the $\mathrm{Na}^{+}$and $\mathrm{Cl}^{-}$. It was heated to $80{ }^{\circ} \mathrm{C}$ for eight hours in an oven to evaporate the hexane. Finally, the iron-oleate complex in a waxy solid form was obtained for further use. The other metal-oleate complexes were prepared in the same way.

\subsection{Synthesis of hydrophobic HAp nanorods}

HAp nanorods were prepared by a hydrothermal method. Firstly, in a $40 \mathrm{~mL}$ Teflon-lined autoclave, $4 \mathrm{~mL}$ oleic acid, $1 \mathrm{~mL}$ oleylamine and $16 \mathrm{~mL}$ ethanol was mixed together to form a homogeneous solution, and an aqueous solution of $\mathrm{Ca}\left(\mathrm{NO}_{3}\right)_{2} \cdot 4 \mathrm{H}_{2} \mathrm{O}(0.25 \mathrm{M}, 7 \mathrm{~mL})$ was added to produce a calciumoleate precursor, then the $\mathrm{Na}_{3} \mathrm{PO}_{4} \cdot 12 \mathrm{H}_{2} \mathrm{O}(0.15 \mathrm{M}, 7 \mathrm{~mL})$ solutions were add in with continued agitation. The mixture was stirred for $5 \mathrm{~min}$, sealed, and hydrothermally treated at $150{ }^{\circ} \mathrm{C}$ for 10 hours. The HAp product was collected by centrifugation and washed with cyclohexane and ethanol several times.

\subsection{Synthesis of $\mathrm{Ca}-\mathrm{PO}_{4}-\mathrm{CO}_{3}$ (CHAp) nanowires}

In a $40 \mathrm{~mL}$ Teflon-lined autoclave, octadecylamine $(0.5 \mathrm{~g})$ was dissolved in $16 \mathrm{~mL}$ ethanol, then $4 \mathrm{~mL}$ oleic acid and aqueous $\mathrm{Ca}\left(\mathrm{NO}_{3}\right)_{2} \cdot 4 \mathrm{H}_{2} \mathrm{O}(0.25 \mathrm{M}, 7 \mathrm{~mL})$ were added in the mixture. Then, an aqueous solutions of $\mathrm{Na}_{2} \mathrm{CO}_{3}(0.25 \mathrm{M}, 7 \mathrm{~mL})$ and $\mathrm{Na}_{3} \mathrm{PO}_{4} \cdot 12 \mathrm{H}_{2} \mathrm{O}(0.15 \mathrm{M}, 3.5 \mathrm{~mL})$ were added in. The mixture was agitated for another $5 \mathrm{~min}$, then sealed and hydrothermally treated at a temperature of $150{ }^{\circ} \mathrm{C}$ for $12 \mathrm{~h}$. The obtained nanowires were collected by centrifugation, washed three times with cyclohexane and ethanol $(\mathrm{v} / \mathrm{v}=1: 2)$ for further ion exchange.

\subsection{Doping HAp or CHAp nanocrystals by postsynthetic ion exchange}

In a flask $500 \mathrm{mg}$ HAp nanocrystals (hydrophobic HAp nanorods; CHAp nanowires), $5 \mathrm{~g}$ oleate and $5 \mathrm{~mL}$ ODE were mixed. It was then treated at a controlled temperature of $100-300{ }^{\circ} \mathrm{C}$ for 1 hour under the protection of argon. After cooled to room temperature naturally, the product was washed and centrifuged with ethanol and cyclohexane for three times. The multi-ion exchange is carried out in the same way. For example, $500 \mathrm{mg}$ hydrophobic HAp nanocrystals with $2.5 \mathrm{~g}$ copper oleate and $2.5 \mathrm{~g}$ zinc oleate were mixed with $5 \mathrm{~mL}$ ODE. It was then treated at a temperature of $200{ }^{\circ} \mathrm{C}$ for 1 hour under the protection of argon. After cooled to room temperature naturally, the product was washed and centrifuged with ethanol and cyclohexane for three times to yield HAp: $\mathrm{Zn}^{2+}, \mathrm{Cu}^{2+}$ nanorods.

\subsection{Co-nucleation doping of $\mathrm{Fe}^{3+}$ with the HAp synthesis}

The process is similar to synthesis of hydrophobic HAp nanorods, firstly, in a $40 \mathrm{~mL}$ Teflon-lined autoclave, $4 \mathrm{~mL}$ oleic acid, $1 \mathrm{~mL}$ oleylamine and $16 \mathrm{~mL}$ ethanol was mixed together to form a homogeneous solution, a certain mount $\mathrm{Fe}\left(\mathrm{NO}_{3}\right)_{3} \cdot 9 \mathrm{H}_{2} \mathrm{O}$ $(0.25 \mathrm{M}, 1.6 \mathrm{~mL})$ and $\mathrm{Ca}\left(\mathrm{NO}_{3}\right)_{2} \cdot 4 \mathrm{H}_{2} \mathrm{O}(0.25 \mathrm{M}, 5.6 \mathrm{~mL})$ was added, then the $\mathrm{Na}_{3} \mathrm{PO}_{4} \cdot 12 \mathrm{H}_{2} \mathrm{O}(0.15 \mathrm{M}, 7 \mathrm{~mL})$ solutions were add in with continued agitation. The mixture was stirred for $5 \mathrm{~min}$, sealed, and hydrothermally treated at $150{ }^{\circ} \mathrm{C}$ for 10 hours. The HAp product was collected by centrifugation and washed with cyclohexane and ethanol for three times.

\subsection{Synthesis of amphiphilic HAp nanocrystals and ion exchange in water}

The method of synthesis amphiphilic HAp is similar to hydrophobic HAp with slight modification. Typically, $0.45 \mathrm{~g}$ 
PEG20000 was dissolved in an aqueous solution of $\mathrm{Ca}\left(\mathrm{NO}_{3}\right)_{2}$ $\cdot 4 \mathrm{H}_{2} \mathrm{O}(7 \mathrm{~mL}, 0.25 \mathrm{M})$, then $7.2 \mathrm{~mL} \mathrm{OA}$ and $16 \mathrm{~mL}$ ethanol was added and mixed. Then $\mathrm{Na}_{3} \mathrm{PO}_{4} \cdot 12 \mathrm{H}_{2} \mathrm{O}(7 \mathrm{~mL}, 0.15 \mathrm{M})$ was added and stirred for $5 \mathrm{~min}$. Finally, the mixture was sealed and reacted at $110{ }^{\circ} \mathrm{C}$ for $10 \mathrm{~h}$. The white precipitate was collected by centrifugation. After washed with cyclohexane and ethanol $(\mathrm{v} / \mathrm{v}=1: 2)$ for twice, it was further washed with water twice for further ion exchange in water. $50 \mathrm{mg}$ HAp and proper amount of $\mathrm{Fe}\left(\mathrm{NO}_{3}\right)_{3} \cdot 9 \mathrm{H}_{2} \mathrm{O}$ was add in a $5 \mathrm{~mL}$ deionized water to form a $0.005,0.01$ or $0.1 \mathrm{M}$ solution, and the mixture was ultrasonic for an hour, then aged for 24 hours. Finally, the HAp was washed with deionized water three times and freeze dried.

\subsection{Hydrophilic treatment of HAp for cytotoxicity assay}

To carry out the cytotoxicity assay experiment, the HAp nanorods were treated to be hydrophilic. Hydrophobic HAp nanorods (undoped HAp nanorods; HAp:Fe ${ }^{3+}$ nanorods postsynthetic ion exchanged at $150{ }^{\circ} \mathrm{C}$ with the doping ratio of $5.1 \%$ ) of $200 \mathrm{mg}$ were dispersed in $10 \mathrm{~mL}$ cyclohexane. Then $20 \mathrm{~mL}$ ethyl acetate and acetonitrile $(\mathrm{v} / \mathrm{v}=1: 1)$ was added. A $15 \mathrm{~mL}$ water solution containing $0.8 \mathrm{~g} \mathrm{NaIO}_{4}$ was added into the former mixture drop by drop while stirring for $2 \mathrm{~h}$. The mixture is divided into two layers, the upper of cyclohexane is colorless and discarded, the bottom is centrifuged, washed with ethanol and water for three times, yielding the hydrophilic HAp that can be dispersed in water.

\subsection{Cytotoxicity assay}

The human cervical carcinoma (HeLa, KeyGEN BioTECH) cells were cultured in Dulbecco's Modified Eagle's Medium (DMEM, KeyGEN BioTECH) with fetal bovine serum (10\%, FBS, Gibco), penicillin $\left(80 \mathrm{U} \mathrm{mL}^{-1}\right)$, and streptomycin $\left(0.08 \mathrm{mg} \mathrm{mL}^{-1}\right)$ at $37{ }^{\circ} \mathrm{C}$ with $5 \% \mathrm{CO}_{2}$. The cytotoxicity of HAp nanocrystals to HeLa cells was studied by using a LDH-Cytotoxicity Colorimetric Assay Kit (BioVision). HeLa cells were cultured in 96-well plates for $24 \mathrm{~h}$, and the culture medium was discard. Afterwards, $200 \mu \mathrm{L}$ of DMEM medium (without FBS) containing different concentrations $\left(20,40,80,160\right.$, and $\left.320 \mu \mathrm{g} \mathrm{mL}{ }^{-1}\right)$ of HAp nanocrystals was added into corresponding wells, respectively. Cells cultured in DMEM medium (no FBS) were set as low control, and cells in DMEM medium (no FBS) containing 1\% Triton X-100 served as high control. After $24 \mathrm{~h}, 100 \mu \mathrm{L}$ of supernatant from each well was pipetted to another well and mixed with $100 \mu \mathrm{L}$ of the reaction mixture (containing $2.2 \mu \mathrm{L}$ catalyst solution and $97.8 \mu \mathrm{L}$ dye solution, BioVision) was added into each well. After being incubated at room temperature for $20 \mathrm{~min}$ away from light, the samples were measured using a microtiter plate reader under the wavelength of $490 \mathrm{~nm}$ (PowerWave $\mathrm{XS}_{2}$, BioTek). The percentage of cell viability was calculated using the formula as follows: cell viability $=100 \%-$ (test sample - low control)/(high control - low control) $\times$ $100 \%$.

\subsection{Characterization}

The power X-ray diffraction (XRD) patterns were recorded using a D/max $2500 \mathrm{VL} / \mathrm{PC}$ diffractometer equipped with graphite monochromatized $\mathrm{Cu} \mathrm{K} \alpha$ radiation $(\lambda=0.15406 \mathrm{~nm})$, the samples were ground into powder and measured in a voltage of $40 \mathrm{kV}$ at a rate of $2^{\circ} \mathrm{min}^{-1}$ and angle range of $20-60^{\circ}$. The phase was identified by comparison to the standard HAp X-ray diffraction pattern. The morphologies and sizes of the obtained samples were observed with a JEM-200CX transmission electron microscope (TEM), with accelerating voltage of $80 \mathrm{kV}$. The functional groups of the samples were characterized using a Fourier-transform infrared spectrophotometer (Vector 22 spectrometer) with the $\mathrm{KBr}$ pellet technique, FTIR spectra were the range of $4000-400 \mathrm{~cm}^{-1}$. The elemental analysis of the samples was determined by energy dispersive spectrometer (EDS). The high-resolution transmission electron microscopy (HRTEM) images were taken on JEOL-2100F with accelerating voltage of $200 \mathrm{kV}$. Inductively coupled plasma (ICP) measurements were performed on an induction coupled plasma quantometer (Jarrell-Ash $1100+2000)$.

\section{Results and discussion}

\subsection{Postsynthetic doping mechanism}

A schematic illustration of the doping mechanism is shown in Fig. 1. Briefly, the un-doped HAp nanocrystals were firstly synthesized, washed and purified. Then the doping processed is performed in the organic solvent with a high boiling point such as octadecene (ODE). The doping metal is introduced in the organic soluble form, e.g. metal oleate. No water is introduced or formed during the doping. Therefore the doping temperature can be freely tuned below the thermal decomposing of the metal oleate. The doping ions first exchange with the $\mathrm{Ca}^{2+}$ at the surface of HAp nanocrystals and then diffuse into the crystal lattice of HAp.

Fig. 2a shows typical pure and doped HAp nanocrystals. They are well dispersed in cyclohexane, forming transparent solutions with various colors which are from the doped ions. It is worth noting that the color of the metal ions may also affected by the coordination field. For the $\mathrm{Fe}^{3+}$ doped HAp, the surface $\mathrm{Fe}^{3+}$ could be modified by oleic acid; while $\mathrm{Fe}^{3+}$ inside the HAp is thermodynamically preferred to occupy the CaI position than the CaII position. ${ }^{34}$ The color deepens with the doping ratio increases, e.g. $\mathrm{Fe}^{3+}$ doping in this report and $\mathrm{Co}^{2+}$ doped HAps. ${ }^{35}$ Transmission electron microscope (TEM) measurement shows

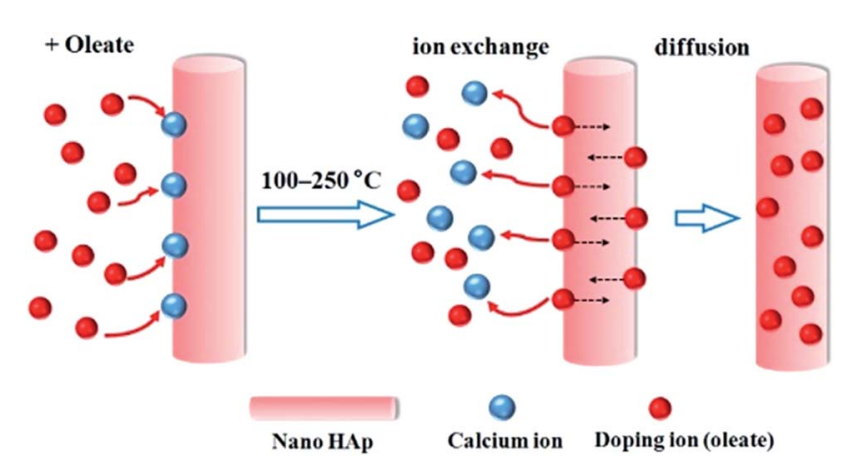

Fig. 1 A schematic illustration of the metal ion doping mechanism for HAp nanocrystals. 
(a)

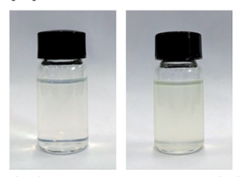

(b)

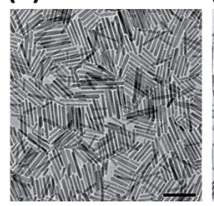

(f)

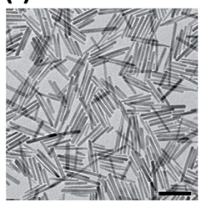

(c)

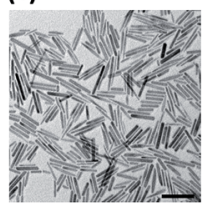

(g)

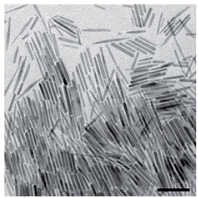

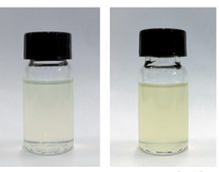

(d)
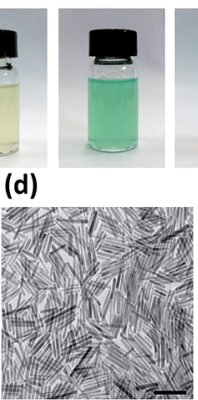

(h)

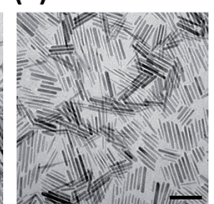

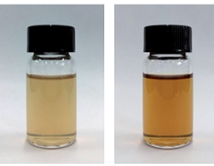

(e)

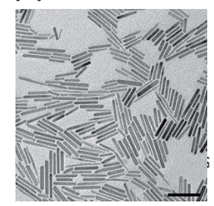

(i)

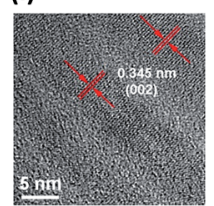

Fig. 2 (a) From left to right the undoped, $\mathrm{Mg}^{2+}, \mathrm{Sr}^{2+}, \mathrm{Zn}^{2+}, \mathrm{Cu}^{2+}, \mathrm{Mn}^{2+}$ and $\mathrm{Fe}^{3+}$ doped HAp nanocrystals, respectively. Their corresponding TEM images $(b-h)$, and (i) a typical high-resolution transmission electron microscopy (HRTEM) image of the undoped HAp nanocrystals, showing the nanocrystals growth along the [002] orientation. ((b-h) Scale bar: $100 \mathrm{~nm})$.

(a)

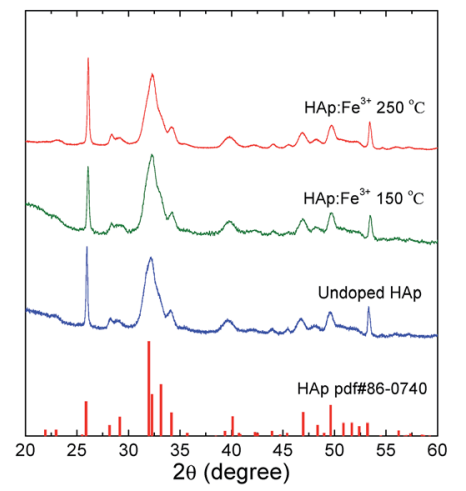

(b)

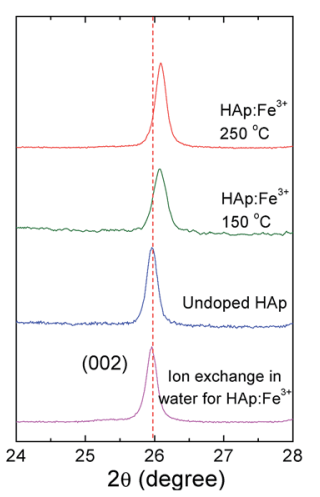

Fig. 3 (a) The XRD patterns of the undoped and $\mathrm{Fe}^{3+}$ doped HAp nanocrystals. (b) The enlarged XRD patterns with the $2 \theta$ between $24^{\circ}$ to $28^{\circ}$ of the corresponding samples.

that the nanorod morphology is preserved for all the HAp nanocrystals doped with metal ions (Fig. 2b-i). The EDS spectra indicate the HAp nanocrystals are successfully doped with metal ions (Fig. S1 $\dagger$ ).

To investigate the doping mechanism, the $\mathrm{Fe}^{3+}$ doped HAp (abbreviated as HAp: $\mathrm{Fe}^{3+}$ ) nanocrystals was selected as a representative prototype. Fig. 3 shows the XRD patterns of the undoped and $\mathrm{Fe}^{3+}$ doped HAp nanocrystals at different temperatures. The XRD peaks of the undoped HAp nanocrystals match well with that of the standard HAp (PDF \#86-0740), indicating the hexagonal crystal structure belonging $P 6_{3} / \mathrm{m}$ space group. The crystal structure of the doped HAp remains the unchanged. But a shift to the higher angle side was observed for the HAp samples doping at elevated temperature of 150 and $250^{\circ} \mathrm{C}$ (Fig. 3). It is consistent with the fact that the radius of the

$\mathrm{Fe}^{3+}$ is significantly smaller than that of $\mathrm{Ca}^{2+}$. So the ion exchange of $\mathrm{Ca}^{2+}$ with $\mathrm{Fe}^{3+}$ leads to the shrinking of the HAp crystal lattice, which is proportional to the doping temperature. It should be mentioned that when the temperature further increased to $280-300{ }^{\circ} \mathrm{C}$, the $\mathrm{Fe}$-oleate will decompose to yield $\mathrm{Fe}_{3} \mathrm{O}_{4}$ nanocrystals, which is proved by TEM and XRD (Fig. S2 $\dagger$ ).

The FTIR spectra of the undoped and $\mathrm{Fe}^{3+}$ doped HAp nanocrystals were measured (Fig. S3 $\uparrow$ ). The band at $3570 \mathrm{~cm}^{-1}$ is the vibration of $\mathrm{OH}$ group in HAp. The bands at 2922 and $2852 \mathrm{~cm}^{-1}$ are assigned to the $\mathrm{CH}_{3}$ and $\mathrm{CH}_{2}$ groups, indicating the surface modification of oleate ligands, which enables the dispersibility of the HAp nanocrystals in nonpolar solvent. The peaks at around 1554 and $1458 \mathrm{~cm}^{-1}$ are assigned to the $\mathrm{COO}^{-}$group of surface oleates. ${ }^{36}$ The intense bands at 1095 and $1027 \mathrm{~cm}^{-1}$ are attributed to the $\mathrm{P}-\mathrm{O}$ asymmetric stretching vibration modes, and peaks at 603 and $562 \mathrm{~cm}^{-1}$ correspond to the O-P-O bending modes. The $\mathrm{Fe}^{3+}$ doped and undoped HAp nanocrystals have almost the same FTIR vibration modes within $4000-400 \mathrm{~cm}^{-1}$, indicating that the crystal structure remained unchanged and the $\mathrm{CH}_{3}, \mathrm{CH}_{2}$, $\mathrm{COO}-$, $\mathrm{P}-\mathrm{O}$ and $\mathrm{O}-\mathrm{P}-\mathrm{O}$ vibration modes can hardly affected by the doped $\mathrm{Fe}^{3+}$. This shielding effect was generally observed in metal doped HAps, including $\mathrm{Fe}^{3+}$ doped HAps. ${ }^{33}$

\subsection{Postsynthetic doping in organic and aqueous solutions}

To understand the difference of the postsynthetic ion exchange performed in organic solvent and in aqueous solution, the HAp nanocrystals doped with $\mathrm{Fe}^{3+}$ were studied. Fig. 4a-d show typical TEM images of undoped and $\mathrm{Fe}^{3+}$ doped HAp nanocrystals with an increasing doping ratio, which is defined by the mole ratio of $\mathrm{Fe}^{3+} /\left(\mathrm{Fe}^{3+}+\mathrm{Ca}^{2+}\right)$. The doping ratio increased monotonically with the doping temperature, e.g. $150{ }^{\circ} \mathrm{C}, 200{ }^{\circ} \mathrm{C}$ and $250{ }^{\circ} \mathrm{C}$ yielding $5.1 \%, 9.1 \%$ and $20.5 \%$, respectively (Fig. $4 \mathrm{~b}-\mathrm{d}$ ). For the sample doped at $150{ }^{\circ} \mathrm{C}$, the same as HAp synthesis temperature, the morphology and size show almost no change (Fig. 4b). In the ODE solution, the $\mathrm{Ca}^{2+}$ on the HAp surface exchanged with $\mathrm{Fe}^{3+}$. Since the concentration of $\mathrm{Fe}^{3+}$ is

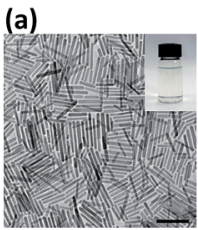

(e)

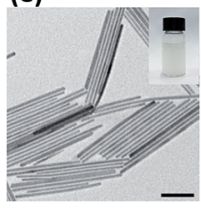

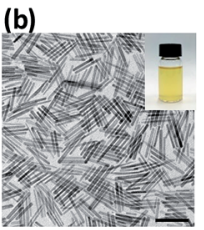

(f)

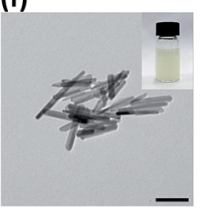

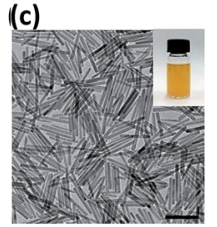

(g)

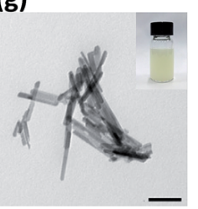

(d)

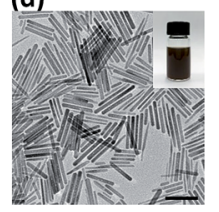

(h)

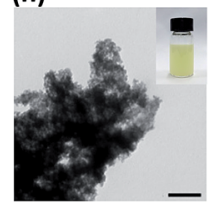

Fig. 4 TEM images of (a) the undoped HAp nanocrystals, and HAp: $\mathrm{Fe}^{3+}$ nanocrystals with the doping ratio of (b) $5.1 \%$, (c) $9.1 \%$, (d) $20.5 \%$. (e) Amphiphilic HAp nanocrystals, and ion exchanged with $\mathrm{Fe}\left(\mathrm{NO}_{3}\right)_{3}$ aqueous solutions yielding the HAp with the doping ratio of (f) $2.3 \%$, (g) $5.8 \%$ and (h) $11.7 \%$. The insets are images of the sample dispersed in cyclohexane $(a-d)$ and in water $(e-h)$. ((a-h) Scale bar: $100 \mathrm{~nm})$. 
relatively high in solution, the surface $\mathrm{Ca}^{2+}$ was effectively exchanged, and $\mathrm{Ca}^{2+}$ vacancies on the surface may also eliminated by $\mathrm{Fe}^{3+}$. To reduce the chemical potential gradient, the $\mathrm{Fe}^{3+}$ ions diffuse from the surface into the nanocrystal, i.e. exchanged with the $\mathrm{Ca}^{2+}$ ions inside the nanocrystal. The diffusion rate increases at elevated temperature. The mass transfer may also happen between HAp nanocrystals during the doping procedure, known as the Ostwald ripening effect. ${ }^{37}$ However, the Ostwald ripening effect has minor impact on the overall morphology mutation and the rod shape retained. At higher doping temperature up to $250{ }^{\circ} \mathrm{C}$, it undergoes only $\sim 16 \%$ and $\sim 8 \%$ increase of the HAp average diameter and length, respectively (Fig. 4d).

To illustrate the advantage of this doping strategy, the comparison is carried out with the $\mathrm{Fe}^{3+}$ ion exchange in water. The $\mathrm{Fe}\left(\mathrm{NO}_{3}\right)_{3}$ aqueous solutions with concentration values of 0 , 0.005, 0.01 and $0.1 \mathrm{M}$ were used, with according ion exchange results shown in Fig. $4 \mathrm{e}-\mathrm{h}$, respectively. It presents that the doping ratio increases monotonically with the $\mathrm{Fe}\left(\mathrm{NO}_{3}\right)_{3}$ concentration. But at the same time, the nanorod morphology becomes out of shape and aggregation takes place. At $\mathrm{Fe}\left(\mathrm{NO}_{3}\right)_{3}$ concentration of $0.1 \mathrm{M}$, the doping ratio amounts to $11.7 \%$, while the HAp nanocrystals completely degrade to amorphous aggregates and the XRD revealed a total crystal decomposing (Fig. S4†). This is due to the fact that HAp is sensitive to the acidity of the aqueous solution induced by the hydrolysis of $\mathrm{Fe}^{3+}$. The HAp sample ion exchanged with $\mathrm{Fe}\left(\mathrm{NO}_{3}\right)_{3}$ aqueous solution of $0.01 \mathrm{M}$ has a doping ratio of $5.8 \%$, comparable to the ODE solution ion exchanged sample of the doping ratio of $5.1 \%$. However, the XRD measurement indicates that the former has no detectable shift of the XRD peak of $(002)$ at $25.97^{\circ}$, while the later shifts to $26.07^{\circ}$ (Fig. 3b). This difference may suggest that for the organic solution doping method, the $\mathrm{Fe}^{3+}$ ions diffuse effectively into the HAp nanocrystals. It could be anticipated that the body doping may have a lower release ratio of the doped ions in biological serving conditions than those of the surface adsorbed ions. On one hand, for the biologically toxic ions, the body doping would reduce the toxicity. On the other hand, for the bio-active or life nutritious doped ions, a long term releasing could be expected.

\subsection{Cytotoxicity assay}

The cytotoxicity assay was performed to study the undoped and doped HAp nanocrystals by using the Lactate Dehydrogenase (LDH) cytotoxicity assay. The human cervical carcinoma (HeLa) cells were cultured for $24 \mathrm{~h}$ at $37^{\circ} \mathrm{C}$ in cell media containing 0 $320 \mu \mathrm{g} \mathrm{mL}{ }^{-1}$ of HAp: $\mathrm{Fe}^{3+}$ nanocrystals with the doping ratio of $5.1 \%$ (postsynthetic ion exchanged at $150{ }^{\circ} \mathrm{C}$ ), and the undoped HAp nanocrystals with the same size and morphology served as a control experiment (Fig. S5 $\dagger$ ). Both of the HeLa cells treated with undoped HAp and HAp: $\mathrm{Fe}^{3+}$ nanocrystals show nearly $100 \%$ viability throughout the $0-320 \mu \mathrm{g} \mathrm{mL}^{-1}$ range, indicating their excellent biocompatibility.

\subsection{Co-nucleation doping and postsynthetic doping}

In the investigation of co-nucleation doping, the $\mathrm{Fe}^{3+}$ ions were introduced into the synthesis of HAp nanocrystals at the beginning of the reaction. It turned out that the resulting product has diverse morphologies (Fig. S6†). The doping of $\mathrm{Ca}-$ $\mathrm{PO}_{4}-\mathrm{CO}_{3}\left(\mathrm{CHAp},{ }^{14}\right)$ solid-solution nanowires with $\mathrm{Fe}^{3+}$ by the postsynthetic ion exchange also indicates that the method is very effective (Fig. S7†). Furthermore, and bimetallic ions codoping HAp: $\mathrm{Zn}^{2+}, \mathrm{Cu}^{2+}$ nanocrystals were also successfully achieved by this method (Fig. S8†). These results further demonstrate the superiority and generality of the postsynthetic organic solution doping strategy.

\subsection{The maximum doping ratio}

The maximum of the HAp doping ratio depends on several factors. First, the doping should not destroy or change the crystal structure. So the maximum doping ratio various for different doping metals. The limit is also related the HAp size, since the surface atom ratio and specific surface energy increase with the HAp size decreasing. Given a closed reaction system, the maximum doping ratio is achieved at the chemical equilibrium point, when the Gibbs free energy change is zero:

$$
\Delta G=0
$$

and $\Delta G$ can be written as:

$$
\begin{gathered}
\Delta G=\mathrm{RT} \ln \left(Q / K^{\theta}\right) \\
Q \propto 1 /\left[\mathrm{C}_{\mathrm{M}}\right]
\end{gathered}
$$

where $Q$ is reaction quotient; $K^{\theta}$ is the standard equilibrium constant; $\left[\mathrm{C}_{\mathrm{M}}\right]$ is the concentration of the doping ions in solution. From the equilibrium state, increasing the $\left[\mathrm{C}_{\mathrm{M}}\right]$ makes $\Delta G<0$. It will drive for further doping. If the starting $\left[\mathrm{C}_{\mathrm{M}}\right]$ is high enough, the doping level can keep rising until the HAp structure limit. However, the $\left[\mathrm{C}_{\mathrm{M}}\right]$ has a maximum value in the state of the pure precursor. Therefore, the doping maximum depends on the lower value from the doping precursor limit and the structure controlled limit. The chemical equilibrium constant of the doping reaction can be tuned by the temperature:

$$
\ln \left({K_{1}}^{\theta} / K_{2}{ }^{\theta}\right)=-\Delta H_{\mathrm{r}}{ }^{\theta}\left(1 / T_{1}-1 / T_{2}\right) / R
$$

where $\Delta H_{\mathrm{r}}{ }^{\theta}$ is the standard enthalpy change of the doping; $R$ is the gas constant. In case the doping reaction is endothermic:

$$
\Delta H_{\mathrm{r}}^{\theta}>0
$$

higher doping level can be achieved in higher temperature. This is experimentally supported in this work. At the same time, the doping procedure is faster at higher temperature and/or higher doping ion concentration in solution.

\section{Conclusion}

In conclusion, a general postsynthetic ion exchange method was developed for doping of various metal ions into the HAp nanocrystals in organic solvent. This method is featured in not only its tunable doping ratio (for $\mathrm{Fe}^{3+}$ it is $0-20 \%$, higher than that ion exchanged in aqueous solution), but also the preserved 
nanocrystal morphology, size, crystal structure, pure products and effective body doping. The merits of this technique will contribute to the applications of the doped functional HAp nanocrystals in artificial bones, tissue engineering, drug delivery and biomarker. The only obstacle is that organic solvents and precursors are involved in this method. It may hinder direct biomedical use. The surface of HAps needs to be treated as hydrophilic and biocompatible, as we did for the cell experiment. To carry out the doping in biocompatible none aqueous solvent is on going. We expect that it may further be applied to HAp anion doping, such as $\mathrm{F}^{-}, \mathrm{CO}_{3}{ }^{2-}$ or $\mathrm{SeO}_{3}{ }^{2-}$ etc., and extend to the doping of other nanocrystals.

\section{Conflicts of interest}

There are no conflicts to declare.

\section{Acknowledgements}

This work was supported by the National Natural Science Foundation of China (NSFC, 51572120, 21376190, 21676215, 21533012 and 51503101), China Postdoctoral Science Foundation (2013M542378, 2014T70933), Shaanxi Provincial Postdoctoral Science Special Foundation (No. [2014]907), Shaanxi Provincial Science Foundation (2014SZS07-Z02, 2017SF-201), Scientific Research Program of Shaanxi Provincial Department of Education (15JS104), Six Talent Peaks Project in Jiangsu Province No. 184080H102231, and Nanjing Normal University Young Leading Talent Fund No. 184080H20210.

\section{References}

1 J. Hui and X. Wang, Inorg. Chem. Front., 2014, 1, 215-225.

2 S. V. Dorozhkin, Int. J. Chem. Mater. Sci., 2013, 1, 105-174.

3 K. Lin, C. Wu and J. Chang, Acta Biomater., 2014, 10, 40714102.

4 M. Vallet-Regí and J. M. González-Calbet, Prog. Solid State Chem., 2004, 32, 1-31.

5 C. Heng, X. Zheng, M. Liu, D. Xu, H. Huang, F. Deng, J. Hui, X. Zhang and Y. Wei, Appl. Surf. Sci., 2016, 386, 269-275.

6 Y. Huang, G. Zhou, L. Zheng, H. Liu, X. Niu and Y. Fan, Nanoscale, 2012, 4, 2484-2490.

7 X. Zheng, J. Hui, H. Li, C. Zhu, X. Hua, H. Ma and D. Fan, Mater. Sci. Eng., C, 2017, 75, 699-705.

8 S. V. Dorozhkin, Prog. Biomater., 2016, 5, 9-70.

9 J. Hui, X. Zhang, Z. Zhang, S. Wang, L. Tao, Y. Wei and X. Wang, Nanoscale, 2012, 4, 6967-6970.

10 X. Zheng, M. Liu, J. Hui, D. Fan, H. Ma, X. Zhang, Y. Wang and Y. Wei, Phys. Chem. Chem. Phys., 2015, 17, 20301-20307.

11 X. Zhang, J. Hui, B. Yang, Y. Yang, D. Fan, M. Liu, L. Tao and Y. Wei, Polym. Chem., 2013, 4, 4120-4125.

12 J. Hui and X. Wang, Chem.-Eur. J., 2011, 17, 6926-6930.
13 J. Sun, X. Zheng, H. Li, D. Fan, Z. Song, H. Ma, H. Xiu and J. Hui, Mater. Sci. Eng., C, 2017, 73, 596-602.

14 J. Hui, Q. Yu, Y. Long, Z. Zhang, Y. Yang, P. Wang, B. Xu and X. Wang, Chem.-Eur. J., 2012, 18, 13702-13711.

15 V. Uskoković, M. A. Iyer and V. M. Wu, J. Mater. Chem. B, 2017, 5, 1430-1445.

16 W. Luo, Y. Liu and X. Chen, Sci. China Mater., 2015, 58, 819850.

17 Z. C. Xiong, Y. J. Zhu, F. F. Chen, T. W. Sun and Y. Q. Shen, Chem.-Eur. J., 2016, 22, 11224-11231.

18 J. Hui, G. Xiang, X. Xu, J. Zhuang and X. Wang, Inorg. Chem., 2009, 48, 5614-5616.

19 D. J. Norris, A. L. Efros and S. C. Erwin, Science, 2008, 319, 1776-1779.

20 N. Pradhan, S. D. Adhikari, A. Nag and D. D. Sarma, Angew. Chem., Int. Ed., 2017, 56, 7038-7054.

21 R. Zeng, R. Shen, Y. Zhao, X. Li, Z. Sun and Y. Shen, Nanotechnology, 2014, 25, 135602.

22 G. Galli, Nature, 2005, 436, 32-33.

23 N. Pradhan, D. Goorskey, J. Thessing and X. Peng, J. Am. Chem. Soc., 2005, 127, 17586-17587.

24 V. A. Vlaskin, C. J. Barrows, C. S. Erickson and D. R. Gamelin, J. Am. Chem. Soc., 2013, 135, 14380-14389.

25 G. Nedelcu, L. Protesescu, S. Yakunin, M. I. Bodnarchuk, M. J. Grotevent and M. V. Kovalenko, Nano Lett., 2015, 15, 5635-5640.

26 M. Miyake, K. Watanabe, Y. Nagayama, H. Nagasawa and T. Suzuki, J. Chem. Soc., Faraday Trans., 1990, 86, 2303-2306.

27 J. A. Gómez del Rio, P. J. Morando and D. S. Cicerone, J. Environ. Manage., 2004, 71, 169-177.

28 S. Saxena and S. F. D'Souza, Environ. Int., 2006, 321, 199-202.

29 A. Yasukawa, T. Yokoyama, K. Kandori and T. Ishikawa, Colloids Surf., A, 2007, 299, 203-208.

30 T. Suzuki, T. Hatsushika and Y. Hayakawa, J. Chem. Soc., Faraday Trans., 1981, 77, 1059-1062.

31 A. Bigi, S. Panzavolta and N. Roveri, Biomaterials, 1998, 19, 739-744.

32 D. Laurencin, N. Almora-Barrios, N. H. de Leeuw, C. Gervais, C. Bonhomme, F. Mauri, W. Chrzanowski, J. C. Knowles, R. J. Newport, A. Wong, Z. H. Gan and M. E. Smith, Biomaterials, 2011, 32, 1826-1837.

33 H. R. Low, N. Phonthammachai, A. Maignan, G. A. Stewart, T. J. Basto, L. L. Ma and T. J. White, Inorg. Chem., 2008, 47, 11774-11782.

34 M. Jiang, J. Terra, A. M. Rossi, M. A. Morales, E. B. Saitovitch and D. E. Ellis, Phys. Rev. B: Condens. Matter Mater. Phys., 2002, 66, 224107.

35 K. P. Tank, K. S. Chudasama, V. S. Thaker and M. J. Joshi, J. Nanopart. Res., 2013, 15, 1644.

36 B. Q. Lu, Y. J. Zhu and F. Chen, Chem.-Eur. J., 2014, 20, 12421246.

37 P. W. Voorhees, J. Stat. Phys., 1985, 38, 231-252. 